\title{
The Approximate Solution of 2D Dirichlet Problem in Doubly Connected Domains
}

\author{
Atallah El-shenawy (D) and Elena A. Shirokova \\ Kazan Federal University, Russia \\ Correspondence should be addressed to Atallah El-shenawy; atallahtm@yahoo.com
}

Received 24 January 2018; Revised 5 June 2018; Accepted 20 June 2018; Published 9 July 2018

Academic Editor: Jacopo Bellazzini

Copyright (C) 2018 Atallah El-shenawy and Elena A. Shirokova. This is an open access article distributed under the Creative Commons Attribution License, which permits unrestricted use, distribution, and reproduction in any medium, provided the original work is properly cited.

\begin{abstract}
We propose a new method for constructing an approximate solution of the two-dimensional Laplace equation in an arbitrary doubly connected domain with smooth boundaries for Dirichlet boundary conditions. Using the fact that the solution of the Dirichlet problem in a doubly connected domain is represented as the sum of a solution of the Schwarz problem and a logarithmic function, we reduce the solution of the Schwartz problem to the Fredholm integral equation with respect to the boundary value of the conjugate harmonic function. The solution of the integral equation in its turn is reduced to solving a linear system with respect to the Fourier coefficients of the truncated expansion of the boundary value of the conjugate harmonic function. The unknown coefficient of the logarithmic component of the solution of the Dirichlet problem is determined from the following fact. The Cauchy integral over the boundary of the domain with a density that is the boundary value of the analytical in this domain function vanishes at points outside the domain. The resulting solution of the Dirichlet problem is the sum of the real part of the Cauchy integral in the given domain and the logarithmic function. In order to avoid singularities of the Cauchy integral at points near the boundary, the solution at these points is replaced by a linear function. The resulting numerical solution is continuous in the domain up to the boundaries. Three examples of the solution of the Dirichlet problem are given: one example demonstrates the solution with constant boundary conditions in the domain with a complicated boundary; the other examples provide a comparison of the approximate solution with the known exact solution in a noncircular domain.
\end{abstract}

\section{Introduction}

Here we introduce the Cauchy integral method for the solution of the Dirichlet problem in doubly connected domains. The proposed method gives an analytical approximate solution to this problem. This analytical solution is differentiable at the interior points and can be calculated at any point in the domain of solution and this is the main advantage of the Cauchy integral method. The method can be applied to arbitrary simply and multiply connected domains with smooth boundaries. Furthermore, it is applicable for the domains with piecewise smooth boundary curves which can be approximated by Fourier polynomial. This technic can be extended to apply the method to Poisson equation, biharmonic equation, and some other types of problems with different types of boundary conditions (Neumann and mixed). It can be applied for solving Riemann-Hilbert problem for analytical functions. The base of these problems is the Dirichlet problem. Here we introduce the solution of 2D Laplace equation with Dirichlet conditions in order to declare the basic idea of the method. In Section 3 we have to improve the behavior of the solution in the form of the Cauchy integral with the help of the linearization technic due to the singularity of Cauchy integral at the points closed to the boundaries.

The mathematical theory regarding Laplace's equation is often referred to as the potential theory, given the significance the equation holds for describing physical phenomena such as gravitational and electrical potentials. Laplace equation with Dirichlet boundary conditions arises in different areas such as electrostatics (where it describes the electrostatic potential in a charge-free region), gravitation (where it describes the gravitational potential in free space), steady state flow of inviscid fluids, and steady state heat conduction. Many authors (e.g., [1-4]) introduced integral equation methods for the two-dimensional Laplace equation solution 
in order to calculate the potential field. The boundary value problem was reduced to an integral equation. The singular kernel in the obtained boundary integral equation was manipulated so that the numerical integration methods or any other numerical methods can be applied directly to evaluate the integrals without any difficulty.

The numerical solution of two-dimensional Laplace equation with Dirichlet boundary conditions in doubly connected domain has been introduced by many authors; for example, the complex variable boundary element methods has been presented in [5]. A highly accurate collocation Trefftz method appeared in [6].

The proposed method constructs the approximate Cauchy integral solution of 2D Dirichlet problem in doubly connected domains. The method is based on the reduction of the problem to the Fredholm integral equation of the second kind for the boundary values of the conjugate harmonic function. The singularity of the obtained integral equation is overcome by using the Hilbert formula. The solution of the resulting integral equation is reduced to the solution of a truncated linear system by using the truncated Fourier series. Finally, the solution of Dirichlet problem has the form of the real part of the Cauchy integral. The solution at the points near the boundaries is approximated by linear functions.

\section{2D Dirichlet Problem for the Laplace Equation}

Let $\Omega$ be a doubly connected domain and $\partial \Omega=L_{0} \cup L_{1}$ be the smooth boundary of $\Omega$, where $L_{0}$ is the outer smooth curve and $L_{1}$ is the inner smooth curve. The corresponding Dirichlet problem for the Laplace equation is to find the doubly differentiable in $\Omega$ function $u(x, y)$, which is continuous in $\Omega \cup \partial \Omega$ and satisfies the Laplace equation

$$
\frac{\partial^{2} u(x, y)}{\partial x^{2}}+\frac{\partial^{2} u(x, y)}{\partial y^{2}}=0, \quad(x, y) \in \Omega,
$$

and the boundary conditions in the form

$$
\begin{aligned}
& u(x(t), y(t))= \begin{cases}f_{0}(t), & (x(t), y(t)) \in L_{0} \\
f_{1}(t), & (x(t), y(t)) \in L_{1}\end{cases} \\
& t \in[0,2 \pi] .
\end{aligned}
$$

\section{The Numerical Solution of the 2D Dirichlet Problem in Doubly Connected Domain}

3.1. The Cauchy Integral Method. Consider a doubly connected domain $\Omega, 0 \notin \Omega$ with the boundary $\partial \Omega$. Assume that the boundary is composed of the outer smooth curve $L_{0}$ : $z_{0}(t), t \in[0,2 \pi]$, passed in a counterclockwise direction, and the inner smooth curve $L_{1}: z_{1}(t), t \in[0,2 \pi]$, passed in a clockwise direction. The Dirichlet problem then is as follows: given the functions $f_{j}(t), j=0,1$, it is necessary to find $u(x, y),(x, y) \in \Omega$, such that $f_{j}(t)=\left.u(x, y)\right|_{(x(t), y(t)) \in L_{j}}$, $j=0,1, t \in[0,2 \pi]$.
Assume that $B(z)$ is analytic in $\Omega$; the solution of the Dirichlet problem according to [7] has the form $u(x, y)=$ $\operatorname{Re}(B(z))+A \log |z|, z=x+i y$, where $A$ is a real-valued parameter which will be calculated later. So the boundary values of the analytic in $\Omega$ function $B(z)$ can be written in the parametric form as follows:

$$
\begin{aligned}
B & \left.(z(t))\right|_{\partial \Omega} \\
& = \begin{cases}f_{0}(t)-A \log \left|z_{0}(t)\right|+i g_{0}(t), & z(t) \in L_{0}, \\
f_{1}(t)-A \log \left|z_{1}(t)\right|+i g_{1}(t), & z(t) \in L_{1} .\end{cases}
\end{aligned}
$$

Due to [7] the function $B(x, y)$ is analytic in $\Omega$ if and only if

$$
\begin{aligned}
& f_{k}(t)-A \log \left|z_{k}(t)\right|+i g_{k}(t) \\
& \quad=\sum_{j=0}^{1} \frac{1}{i \pi} \int_{0}^{2 \pi} \frac{f_{j}(\tau)-A \log \left|z_{j}(t)\right|+i g_{j}(\tau)}{z_{j}(\tau)-z_{k}(t)} z_{j}^{\prime}(\tau) d \tau .
\end{aligned}
$$

where the corresponding singular integral at the right-hand side of the relation is a principle value integral and $z_{k}(t)$, $k=\{0,1\}$, are written in the form of truncated Fourier polynomials:

$$
\begin{aligned}
& z_{0}(t)=\sum_{k=-m}^{m} c_{k} e^{i k t}, \quad t \in[0,2 \pi], \\
& z_{1}(t)=\sum_{k=-m}^{m} d_{k} e^{i k t}, \quad t \in[0,2 \pi] .
\end{aligned}
$$

After separating the imaginary part in (4) we obtain the following system of equations:

$$
\begin{aligned}
g_{0}(t)=\frac{1}{\pi} \int_{0}^{2 \pi} g_{0}(\tau)\left[\arg \left(z_{0}(\tau)-z_{0}(t)\right)\right]_{\tau}^{\prime} d \tau+\frac{1}{\pi} \\
\cdot \int_{0}^{2 \pi} g_{1}(\tau)\left[\arg \left(z_{1}(\tau)-z_{0}(t)\right)\right]_{\tau}^{\prime} d \tau-\frac{1}{\pi} \\
\cdot \int_{0}^{2 \pi}\left(f_{0}(\tau)-A \log \left|z_{0}(\tau)\right|\right) \\
\cdot\left[\log \left(z_{0}(\tau)-z_{0}(t)\right)\right]_{\tau}^{\prime} d \tau-\frac{1}{\pi} \\
\cdot \int_{0}^{2 \pi}\left(f_{1}(\tau)-A \log \left|z_{1}(\tau)\right|\right) \\
\cdot\left[\log \left(z_{1}(\tau)-z_{0}(t)\right)\right]_{\tau}^{\prime} d \tau, \\
g_{1}(t)=\frac{1}{\pi} \int_{0}^{2 \pi} g_{0}(\tau)\left[\arg \left(z_{0}(\tau)-z_{1}(t)\right)\right]_{\tau}^{\prime} d \tau+\frac{1}{\pi} \\
\cdot \int_{0}^{2 \pi} g_{1}(\tau)\left[\arg \left(z_{1}(\tau)-z_{1}(t)\right)\right]_{\tau}^{\prime} d \tau-\frac{1}{\pi} \\
\cdot \int_{0}^{2 \pi}\left(f_{0}(\tau)-A \log \left|z_{0}(\tau)\right|\right) \\
\cdot\left[\log \left(z_{0}(\tau)-z_{1}(t)\right)\right]_{\tau}^{\prime} d \tau-\frac{1}{\pi}
\end{aligned}
$$




$$
\begin{aligned}
& \cdot \int_{0}^{2 \pi}\left(f_{1}(\tau)-A \log \left|z_{1}(\tau)\right|\right) \\
& \cdot\left[\log \left(z_{1}(\tau)-z_{1}(t)\right)\right]_{\tau}^{\prime} d \tau .
\end{aligned}
$$

We consider the factor $\left(e^{i \tau}-e^{i t}\right)$ in the expression $\left(z_{s}(\tau)-\right.$ $\left.z_{s}(t)\right), s=0,1$ in order to separate the improper PV integral in the Fredholm equation of the second kind as follows $[8,9]$ :

$$
\begin{aligned}
& \log \left[z_{0}(\tau)-z_{0}(t)\right]=\log (2 i)+\log \left(\sin \left(\frac{\tau-t}{2}\right)\right) \\
& +i \frac{\tau+t}{2} \\
& +\log \left(\sum_{k=1}^{m} c_{k} e^{i k t} \sum_{l=0}^{k-1} e^{i l(\tau-t)}-\sum_{k=1}^{m} c_{-k} e^{-i k \tau} \sum_{l=0}^{k-1} e^{i l(\tau-t)}\right), \\
& \log \left[z_{1}(\tau)-z_{1}(t)\right]=\log (2 i)+\log \left(\sin \left(\frac{\tau-t}{2}\right)\right) \\
& +i \frac{\tau+t}{2} \\
& +\log \left(\sum_{k=1}^{m} d_{k} e^{i k t} \sum_{l=0}^{k-1} e^{i l(\tau-t)}-\sum_{k=1}^{m} d_{-k} e^{-i k \tau} \sum_{l=0}^{k-1} e^{i l(\tau-t)}\right),
\end{aligned}
$$

so (7)-(8) take the form

$$
\begin{aligned}
g_{\mathrm{s}}(t)= & \frac{1}{2 \pi} \int_{0}^{2 \pi} g_{\mathrm{s}}(\tau) d \tau+\frac{1}{\pi} \int_{0}^{2 \pi} g_{\mathrm{s}}(\tau) K_{s}(\tau, t) d \tau \\
& -\frac{1}{2 \pi} \int_{0}^{2 \pi} f_{\mathrm{s}}(\tau) \cot \frac{\tau-t}{2} d \tau \\
& -\frac{1}{\pi} \int_{0}^{2 \pi} f_{\mathrm{s}}(\tau) L_{s}(\tau, t) d \tau, \quad s=0,1 .
\end{aligned}
$$

Here

$$
\begin{aligned}
& K_{0}(\tau, t)=\operatorname{Im}\left[\operatorname { l o g } \left(\sum_{k=1}^{m} c_{k} e^{i k t} \sum_{l=0}^{k-1} e^{i l(\tau-t)}\right.\right. \\
& \left.\left.-\sum_{k=1}^{m} c_{-k} e^{-i k \tau} \sum_{l=0}^{k-1} e^{i l(\tau-t)}\right)\right]_{\tau}^{\prime}, \\
& L_{0}(\tau, t)=\operatorname{Re}\left[\operatorname { l o g } \left(\sum_{k=1}^{m} c_{k} e^{i k t} \sum_{l=0}^{k-1} e^{i l(\tau-t)}\right.\right. \\
& \left.\left.-\sum_{k=1}^{m} c_{-k} e^{-i k \tau} \sum_{l=0}^{k-1} e^{i l(\tau-t)}\right)\right]_{\tau}^{\prime}, \\
& K_{1}(\tau, t)=\operatorname{Im}\left[\operatorname { l o g } \left(\sum_{k=1}^{m} d_{k} e^{i k t} \sum_{l=0}^{k-1} e^{i l(\tau-t)}-\sum_{k=1}^{m} d_{-k} e^{-i k \tau}\right.\right. \\
& \left.\left.\quad \sum_{l=0}^{k-1} e^{i l(\tau-t)}\right)\right]_{\tau}^{\prime},
\end{aligned}
$$

$$
\begin{gathered}
L_{1}(\tau, t)=\operatorname{Re}\left[\operatorname { l o g } \left(\sum_{k=1}^{m} d_{k} e^{i k t} \sum_{l=0}^{k-1} e^{i l(\tau-t)}\right.\right. \\
\left.\left.-\sum_{k=1}^{m} d_{-k} e^{-i k \tau} \sum_{l=0}^{k-1} e^{i l(\tau-t)}\right)\right]_{\tau}^{\prime} .
\end{gathered}
$$

Let us search for the solution of the system of Fredholm integral equations (11) in the form of Fourier series as follows:

$$
g_{s}(t)=\alpha_{s}+\sum_{n=1}^{\infty} \alpha_{s n} \cos (n t)+\beta_{s n} \sin (n t), \quad s=0,1
$$

According to $[8,10],(11)$ has a unique solution if we set the summands

$$
\alpha_{\mathrm{s}}=\frac{1}{2 \pi} \int_{0}^{2 \pi} g_{\mathrm{s}}(\tau) d \tau=0, \quad s=0,1
$$

The solvability of (11) is proved in [8] where the Fourier series solution form of the functions $g_{\mathrm{s}}(t), \mathrm{s}=0,1$, leads us to an infinite linear system of equations which can be reduced to a finite one according to the following lemma.

Lemma 1 (see [8]). Let the numbers $j, p>1$ and $a$ constant $U>0$ exist so that $\left|\partial^{j+p} G(\tau, t) / \partial t^{j} \partial \tau^{p}\right| \leq U$ and the function $Y(t)$ possesses the bounded second derivative: $\left|Y^{\prime \prime}(t)\right|<T$. Then, the approximate solution of the uniquely resolvable Fredholm integral equation of the second kind $X(t)=$ $\int_{0}^{2 \pi} G(\tau, t) X(\tau) d \tau+Y(t)$, where $Y(t)$ is $2 \pi$ periodic and $G(\tau, t)$ is $2 \pi$ periodic with respect to both variables, can be reduced to solution of finite linear system with error estimated by $\mathcal{O}\left(1 / N^{2}\right)$ where $N$ is the finite linear system rank.

According to the previous lemma and $[10,11]$ the unique solution of the second kind Fredholm integral equation system (11) can be expressed in the form of truncated Fourier series with arbitrary summands as follows:

$$
g_{\mathrm{s}}(t)=\sum_{n=1}^{N} \alpha_{\mathrm{s} n} \cos (n t)+\beta_{\mathrm{s} n} \sin (n t), \quad s=0,1,
$$

where $\alpha_{\mathrm{s}}=(1 / 2 \pi) \int_{0}^{2 \pi} g_{\mathrm{s}}(\tau) d \tau=0, s=0,1$. Put relation (15) into (11) and write down the finite linear system in the matrix form as follows $[12,13]$ :

$$
(I-W) \lambda=P+A Q
$$


Here

$\lambda$

$$
\begin{aligned}
& =\left(\begin{array}{c}
\alpha_{0 n} \\
\beta_{0 n} \\
\alpha_{1 n} \\
\beta_{1 n}
\end{array}\right) \text { is the vector of unknown coefficients, } \\
P & =\left(\begin{array}{l}
p_{0 n} \\
\widehat{p}_{0 n} \\
p_{1 n} \\
\widehat{p}_{1 n}
\end{array}\right), \\
Q & =\left(\begin{array}{l}
q_{0 n} \\
\widehat{q}_{0 n} \\
q_{1 n} \\
\widehat{q}_{1 n}
\end{array}\right),
\end{aligned}
$$

are the right-hand side vectors, $I$ is the $4 N \times 4 N$ identity matrix, and $W$ is a $4 N \times 4 N$ coefficient block matrix. The elements of these matrices can be easily calculated by writing formulas (11) into the matrix form.

The solution of the linear system of (16) is the vector with the elements that are linear functions of the parameter $A: g_{s}(t)=\widehat{g_{s}}(t)+A \widetilde{g_{s}}(t)$. Since the point $z=0$ is an exterior point of the domain $\Omega$ and $f_{s}(t)-A \log \left|z_{s}(t)\right|+i\left(\widehat{g_{s}}(t)+\right.$ $\left.A \widetilde{g_{s}}(t)\right), s=\{0,1\}$, are the boundary values of the analytic in $\Omega$ function, the value of $A$ can be calculated from the following formula:

$$
\begin{gathered}
\sum_{s=0}^{1}\left(\int_{0}^{2 \pi}\left[f_{s}(t)-A \log \left|z_{s}(t)\right|+i\left(\widehat{g_{s}}(t)+A \widetilde{g_{s}}(t)\right)\right]\right. \\
\left.\cdot \frac{z_{s}^{\prime}(t)}{z_{s}^{k+1}(t)} d t\right)=0, \quad k=0,1, \ldots
\end{gathered}
$$

We can satisfy (18) for $k=0,1, \ldots, N$, if we apply least square method. Finally, the solution $u(x, y)$ of Dirichlet problem (1)-(2) in the doubly connected domain $\Omega$ can be expressed as

$$
\begin{aligned}
& u(x, y) \\
& =\operatorname{Re}\left(\sum_{j=0}^{1} \frac{1}{2 \pi i} \int_{0}^{2 \pi} \frac{f_{j}(t)-A \log \left|z_{j}(t)\right|+i g_{j}(t)}{z_{j}(t)-(x+i y)} z_{j}^{\prime}(t) d t\right) \\
& \quad+\frac{A}{2} \log \left(x^{2}+y^{2}\right) .
\end{aligned}
$$

3.2. Improving the Approximate Solution at the Points Near Boundaries. Here we introduce a new technic to improve the approximate solution at the points near boundaries. The technic depends on the approximation of the harmonic function solution of Dirichlet problem at these points by linear functions.

Let us define the reference curves:

$$
z_{\varepsilon j}(t)=z_{j}(t)+\gamma_{j} i R_{j} \frac{z_{j}^{\prime}(t)}{\left|z_{j}^{\prime}(t)\right|}, \quad j=0,1,
$$

where $R_{j}, j=0,1$ are the radii of curvature of the boundaries $z_{j}(t), j=0,1, \gamma_{j}<1$. Let the approximate solution at the points of reference curves calculated by the Cauchy integral method be $u_{\varepsilon j}(t), j=0,1$.

Define $\widetilde{\Omega}$ is the domain with boundaries $z_{\varepsilon j}(t)$. The solution of Dirichlet problem at the points of $\Omega \backslash \widetilde{\Omega}$ is approximated with linear functions as follows.

Firstly, for every point $\zeta$, we construct a straight line which is perpendicular to the curve $z_{\varepsilon j}(t)$ and passes through this point. The straight line provides the projections of the point on the curves $z_{j}(t), z_{\varepsilon j}(t)$ which will be defined as $z_{j}\left(t_{0}\right)$, $z_{\varepsilon j}\left(t_{0}\right), j=0,1$.

Secondly, the approximate solution of 2D Dirichlet problem is calculated at this point by substituting in the following linear equation:

$$
\begin{aligned}
u_{\zeta}=\frac{u_{\varepsilon j}\left(t_{0}\right)-f_{j}\left(t_{0}\right)}{z_{\varepsilon j}\left(t_{0}\right)-z_{j}\left(t_{0}\right)}\left(t_{0}-z_{j}\left(t_{0}\right)\right)+f_{j}\left(t_{0}\right), & \\
& j=0,1 .
\end{aligned}
$$

\section{Numerical Examples}

The Cauchy integral method was applied to the 2D Dirichlet problem and highly accurate results for regular and irregular doubly connected domains with smooth boundaries are obtained. Numerical examples are presented to verify the accuracy of the proposed method in the earlier sections.

Example 1. Let us define the doubly connected domain with nonstarlike boundaries as in [10]. The boundaries are composed of the outer and inner parametric curves, respectively, as follows:

$$
\begin{array}{rl}
z_{0}(t)=-0.5+e^{i t}+0.5 e^{2 i t}+0.2 i e^{-2 i t}, \quad t \in[0,2 \pi] \\
z_{1}(t)=0.2 i+0.5 e^{-i t}-0.25 e^{-2 i t}+0.1 i e^{2 i t}, & \\
t & t \in[0,2 \pi]
\end{array}
$$

The method was applied and we use a constant boundary values which equal $f_{0}=1, f_{1}=2$ and the contour plot of the solution is shown in Figure 1. The figure shows that the extreme values of the solution are on the boundaries.

Example 2. Consider the doubly connected domain defined in $[6,14]$ as shown in Figure 2. The boundaries consisted of a complex Epitrochoid as the outer smooth boundary curve given by relation

$$
z_{0}(t)=r_{0} \cos (t)+i r_{0} \sin (t), \quad t \in[0,2 \pi],
$$




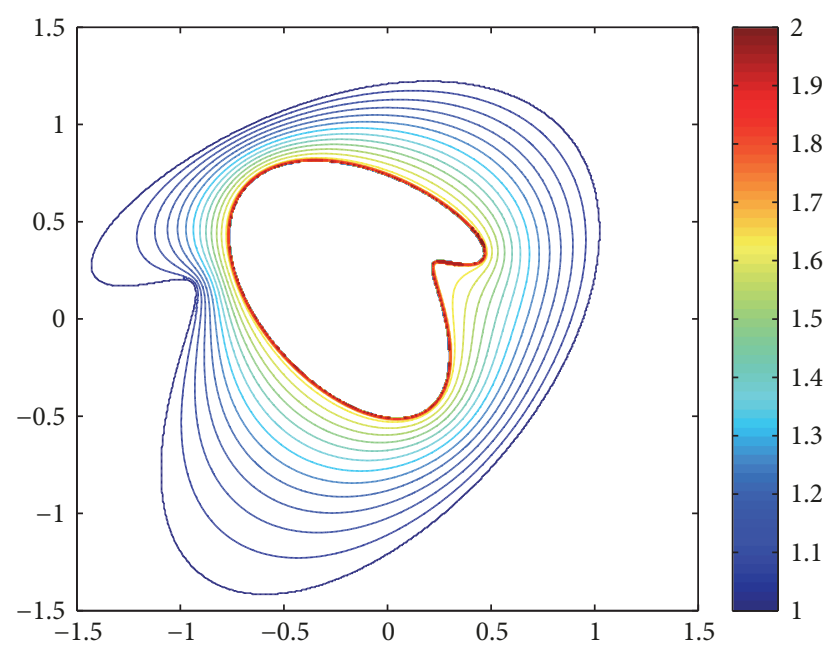

FIGURE 1: The contour plot of the approximate solution with constant boundary conditions in Example 1.

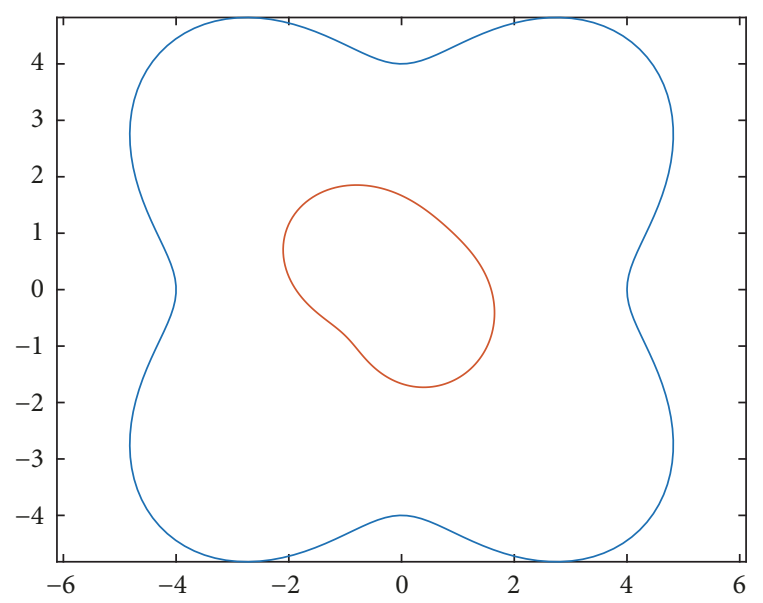

Figure 2: The domain in Example 2.

where

$$
r_{0}(t)=\sqrt{(a+b)^{2}+1-2(a+b) \cos \left(\frac{a t}{b}\right)},
$$

and the inner curve given by $z_{1}(t)=r_{1} \cos (t)+i r_{1} \sin (t), t \in$ $[0,2 \pi]$,

where

$$
r_{1}(t)=\frac{2.5+\cos t+0.5 \sin 2 t}{1.5+0.7 \cos t}
$$

The boundary conditions are derived from the closed form of the exact solution $u(x, y)=e^{x} \cos y$. The method was applied for $\mathrm{a}=4 ; \mathrm{b}=1$ and the value of constant $A$ was calculated and equal to $7.503 E-08$. The absolute error along the points of circle with radius $r=\max _{t \in[0,2 \pi]} r_{1}(t)$ is shown in Figure 3 . The figure demonstrates the accuracy of the method with absolute error less than $1.2325 \times 10^{-5}$ which is better than the Trefftz method introduced in [6]. This example shows the effectivity of our method at the points near the boundaries.

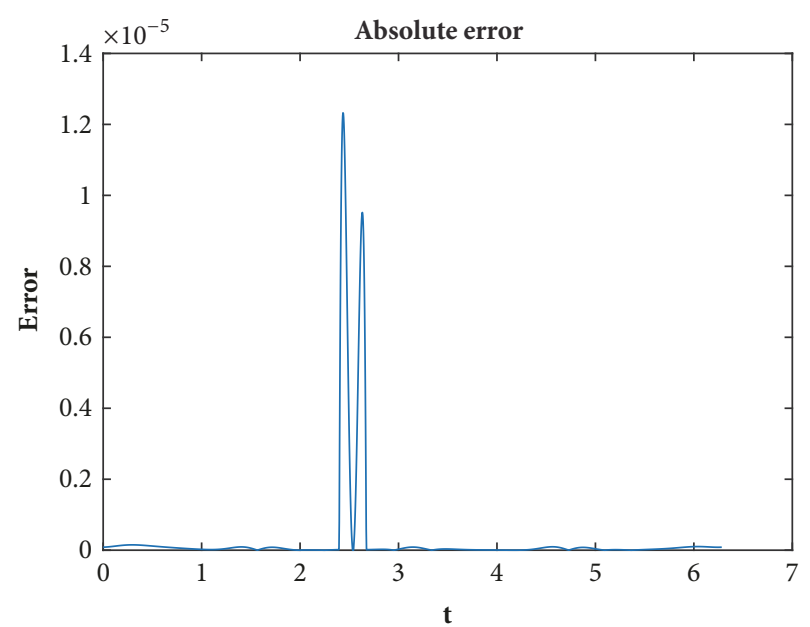

FIgURE 3: The absolute error along circle with radius $\max r_{1}$ in Example 2.

Example 3. Consider the doubly connected domain shown in Figure 4. The boundaries consist of the kite-shape as the outer smooth boundary curve given by

$$
z_{0}(t)=r_{0} \cos (t)+i r_{0} \sin (t), \quad t \in[0,2 \pi],
$$

where

$$
\begin{aligned}
& r_{0}(t) \\
& =1.5 \\
& \quad \times \sqrt{(0.6 \cos t+0.3 \cos 2 t-0.2)^{2}+(0.6 \cos t)^{2}}
\end{aligned}
$$

and the inner curve $z_{1}(t)=r_{1} \cos (t)+i r_{1} \sin (t), t \in[0,2 \pi]$, where

$$
r_{1}(t)=\frac{2.5+\cos t+0.5 \sin 2 t}{1.5+0.7 \cos t}
$$

To test the method we consider the exact solution $u(x, y)=$ $x^{2}-y^{2}$.

Figure 5 shows the absolute error which is calculated along the points of circle with radius equal to 0.6 . The maximum error is $1.315 \mathrm{E}-08$ which shows that the proposed method gives highly accurate results for the interior domain when compared with the exact solution.

\section{Conclusions}

The Cauchy integral method gives highly accurate results for the solution of 2D Dirichlet problem for irregular doubly connected domains. The proposed algorithm improves the approximate solution at the points near the boundaries. The method is applicable for domains bounded by any smooth curve approximated by Fourier polynomial. Numerical experiments are given to verify the efficiency of the method. 


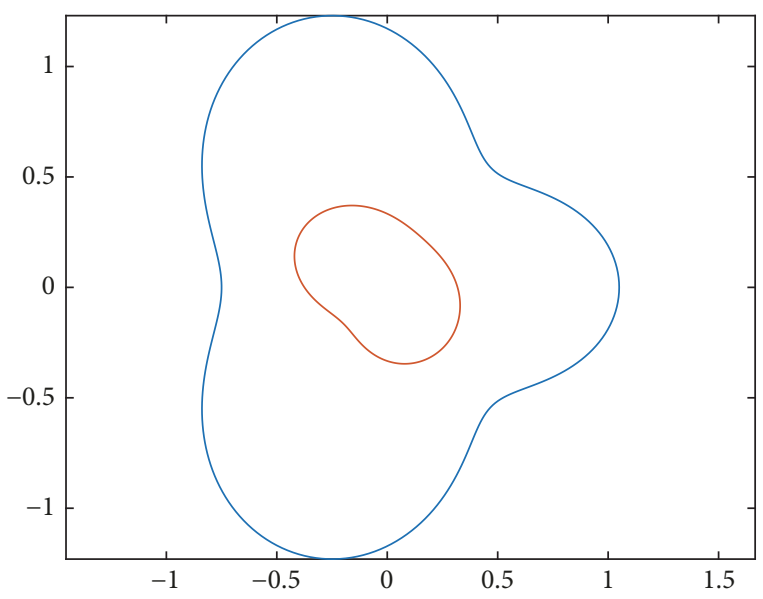

FIGURE 4: Domain in Example 3.

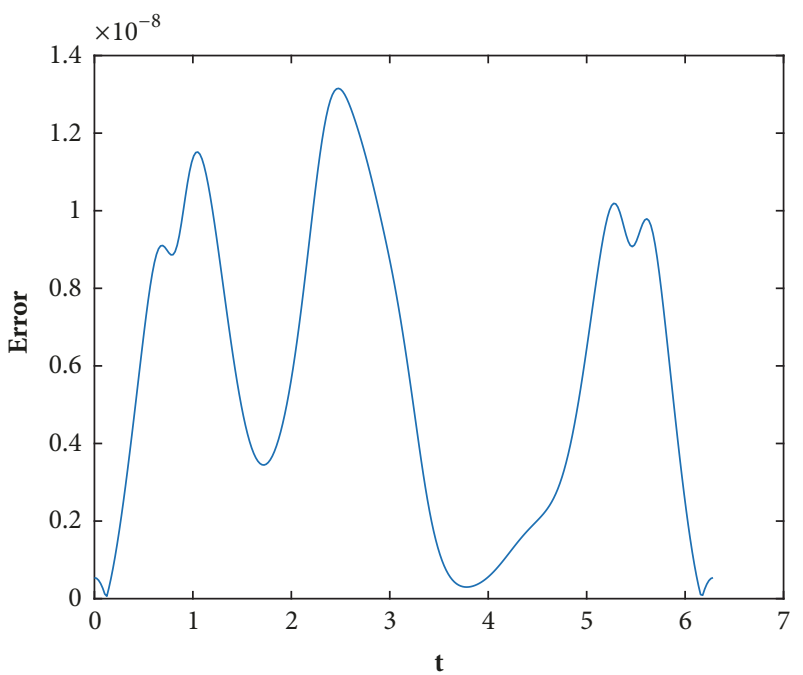

FIgURE 5: The absolute error along a circle with radius 0.6 in Example 3.

\section{Data Availability}

The data used to support the findings of this study are available from the corresponding author upon request.

\section{Conflicts of Interest}

The authors declare that they have no conflicts of interest.

\section{Acknowledgments}

This work is performed according to the Russian Government Program of Competitive Growth of Kazan Federal University.

\section{References}

[1] I. Fredholm, "Sur une classe d'équations fonctionnelles," Acta Mathematica, vol. 27, no. 1, pp. 365-390, 1903.
[2] J. L. Hess and A. M. O. Smith, "Calculation of non-lifting potential flow about arbitrary three-dimensional bodies," Journal of Ship Research, vol. 8, no. 2, pp. 22-44, 1964.

[3] M. A. Jaswon, "Integral equation methods in potential theory," Proceedings of the Royal Society of London. Series A, vol. 275, pp. 23-32, 1963.

[4] J. L. Blue, "Boundary integral solutions of Laplace's equation," Bell Labs Technical Journal, vol. 57, no. 8, pp. 2797-2822, 1978.

[5] C. K. Hsieh and A. J. Kassab, "Complex variable boundary element methods for the solution of potential problems in simply and multiply connected domains," Computer Methods Applied Mechanics and Engineering, vol. 86, no. 2, pp. 189-213, 1991.

[6] C.-S. Liu, "A highly accurate collocation Trefftz method for solving the Laplace equation in the doubly connected domains," Numerical Methods for Partial Differential Equations, vol. 24, no. 1, pp. 179-192, 2008.

[7] F. D. Gakhov, Boundary Value Problems, Oxford: Pergamon Press, 1966.

[8] P. N. Ivanshin and E. A. Shirokova, "Approximate conformal mappings and elasticity theory," Journal of Complex Analysis, Art. ID 4367205, 8 pages, 2016.

[9] N. I. Muskhelishvili, Singular integral equations, Springer Netherlands, Netherlands, 1958.

[10] D. F. Abzalilov and E. A. Shirokova, "The approximate conformal mapping onto simply and doubly connected domains," Complex Variables and Elliptic Equations. An International Journal, vol. 62, no. 4, pp. 554-565, 2017.

[11] E. A. Shirokova, "On the approximate conformal mapping of the unit disk on a simply connected domain," Russian Mathematics (Iz. VUZ), vol. 58, no. 3, pp. 47-56, 2014.

[12] F. G. Tricomi, Integral Equations, Interscience, New York, NY, USA, 1957.

[13] R. G. Cooke, Infinite Matrices \& sequence spaces, Dover publications Inc, New York, NY, USA, 2014.

[14] Z.-Q. Wang, S. Li, Y. Ping, J. Jiang, and T.-F. Ma, "A highly accurate regular domain collocation method for solving potential problems in the irregular doubly connected domains," Mathematical Problems in Engineering, Art. ID 397327, 9 pages, 2014. 


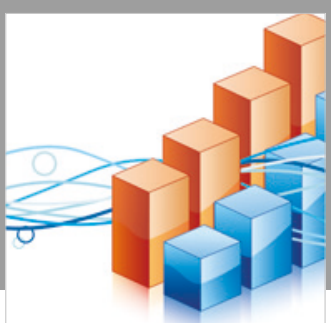

Advances in

Operations Research

\section{-n-m}
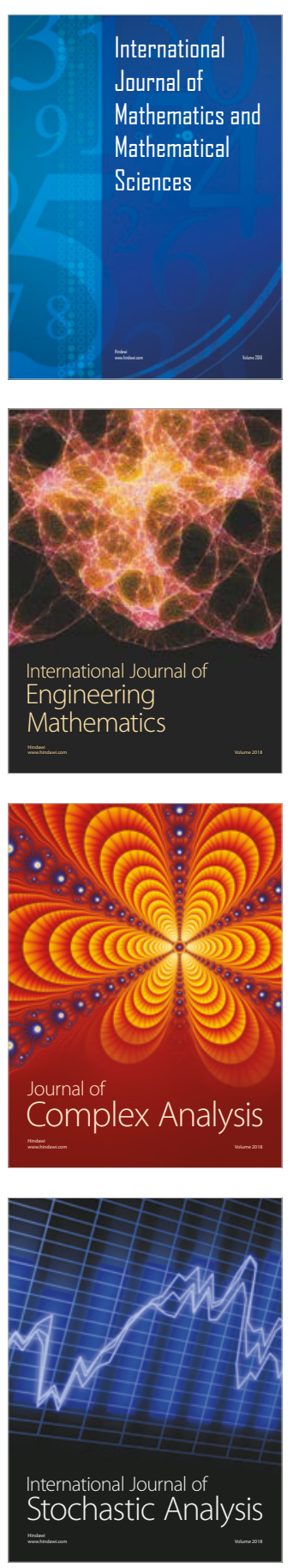
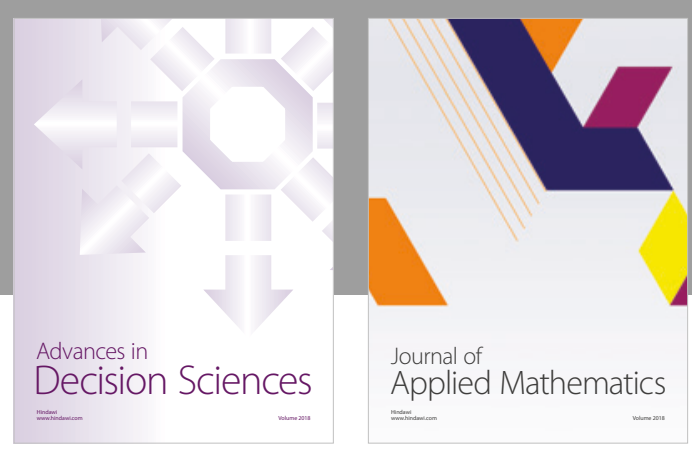

Journal of

Applied Mathematics
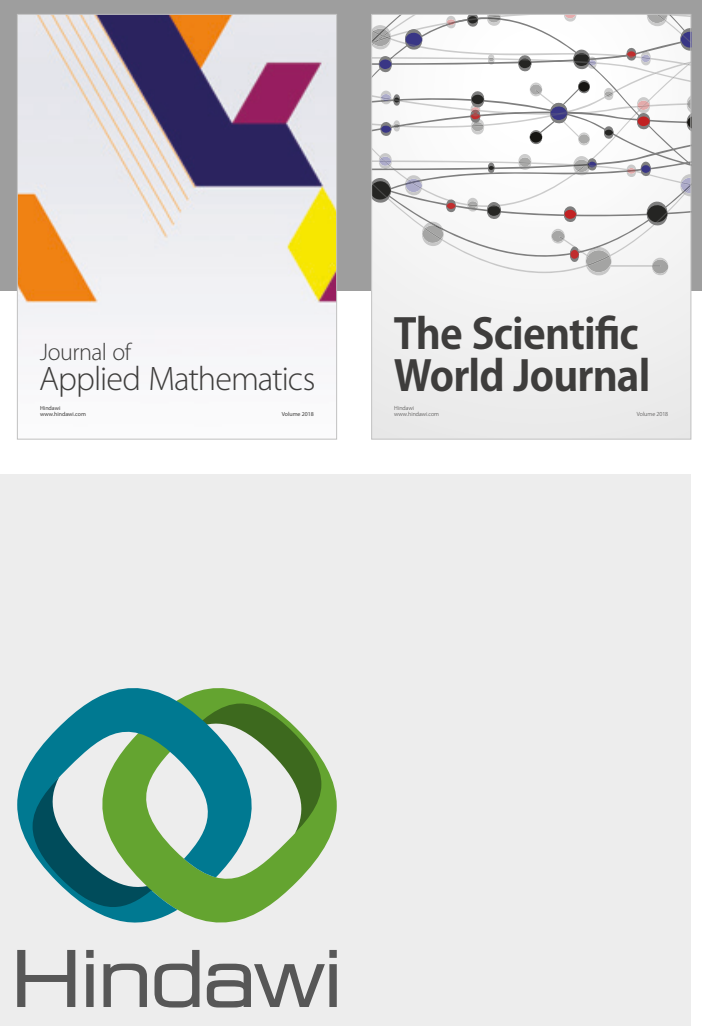

Submit your manuscripts at

www.hindawi.com

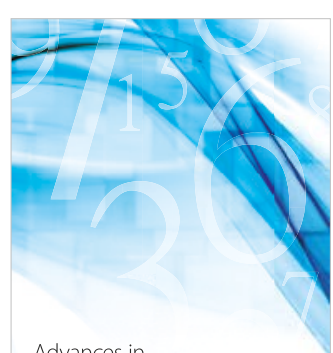

Advances in
Numerical Analysis
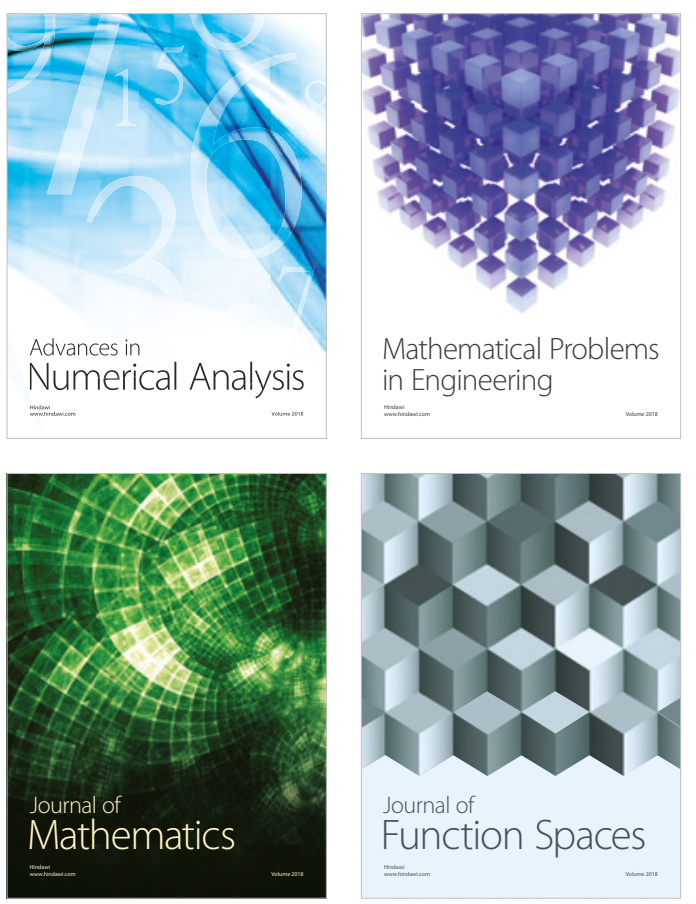

Mathematical Problems in Engineering

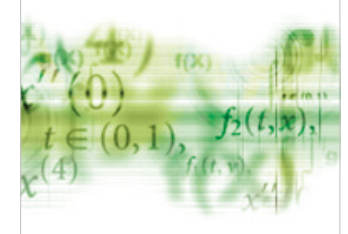

International Journal of

Differential Equations

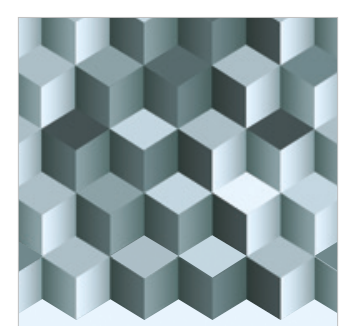

Journal of

Function Spaces

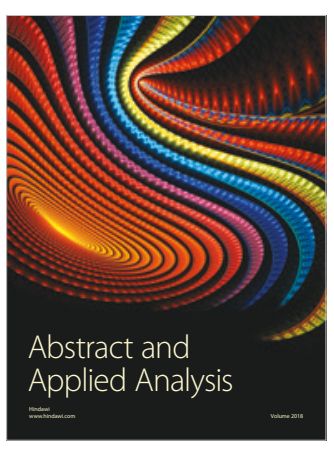

The Scientific

World Journal

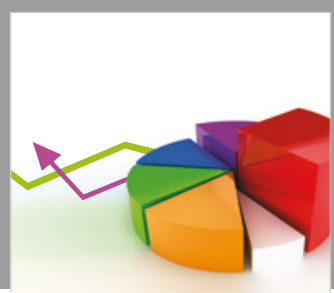

Journal of

Probability and Statistics
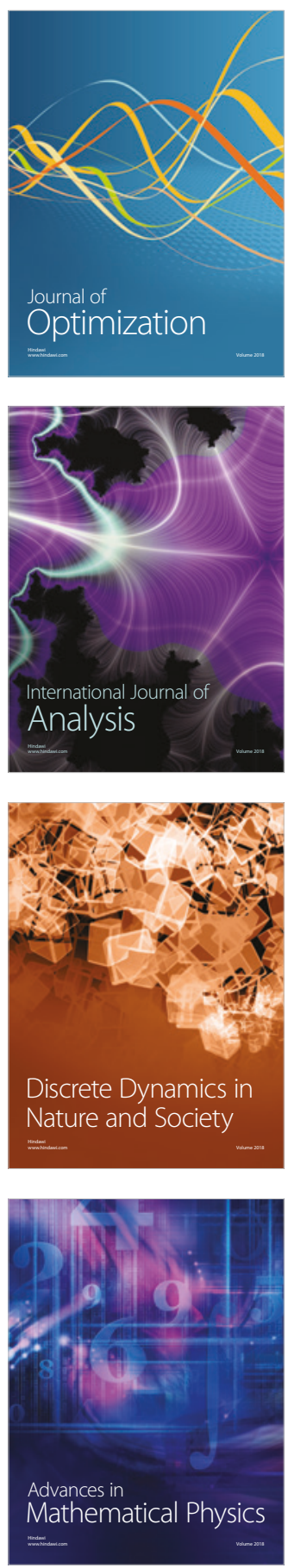